\title{
COVID-19 and Elevated D-Dimer: A Tale of Caution
}

$\mathrm{J}$ Gen Intern Med

DOI: $10.1007 / \mathrm{s} 11606-021-07280-9$

(C) The Author(s) under exclusive licence to Society of General Internal Medicine 2021
$\mathrm{D}$ ear editor:

We read with interest the article written by GarcíaCervera et al. with the analysis of the SEMI-COVID-19 registry with the finding of an admission D-dimer $>3.0 \mu \mathrm{g} / \mathrm{mL}$ as a strong predictor for venous thromboembolism (VTE) in patients hospitalized with COVID-19. ${ }^{1}$ The authors conclude and recommend that this cut-off value ought to prompt the consideration to screen for VTE and initiate full-dose anticoagulation.

We would like to share some thoughts on this matter. In our institution, an observational study was conducted of 3678 patients admitted with COVID-19 in Ohio and Florida between March 13, 2020, and May 13, 2020; 892 (24.3\%) were hospitalized and $296(8.0 \%)$ required admission to the intensive care unit (ICU). Acute thrombotic events occurred in 46 $(5.2 \%)$ of the hospitalized patients and $26(8.8 \%)$ of the ICU patients. The blood D-dimer concentration was elevated in almost all patients admitted with COVID-19, without a distinction between the patients who developed acute thrombosis and those who did not. ${ }^{2}$ Furthermore, the RIETE registry has determined a cut-off value of D-dimer elevation $>20$-fold of normal values to represent an inflection point in patients with COVID-19 to escalate anticoagulation above a thromboprophylaxis dose. $^{3}$

Based on the above, our concern is using D-dimer alone as a decision tool is not sufficient to consider full-dose anticoagulation in the absence of objective documentation of an acute thrombotic event, especially since this practice exposes patients to additional dangers including bleeding. In an observational study from Northwestern University of 1716 adult patients with acute COVID-19 admitted to the hospital, the initiation of therapeutic anticoagulation was associated with an increased risk of adverse outcomes: death (OR 5.93; $95 \%$ CI 3.71-9.47), critical illness (OR 14.51; 95\% CI 7.43-28.31), mechanical ventilation (OR 11.22; 95\% CI 6.67-18.86), and death after first having critical illness (OR 5.51; 95\% CI 2.80

Received August 5, 2021

Accepted November 10, 2021
10.87). Among patients who continued pre-existent anticoagulation, there were no increased adverse outcomes. ${ }^{4}$

Recent analysis of the randomized trials REMAP-CAP, ATTACC, and ACTIV-4a evaluated the clinical outcomes with different strategies of anticoagulation using heparinoids in critically ill patients ( 545 patients with prophylactic dosage vs. 529 patients with therapeutic dose); there was neither a meaningful difference in overall organ support nor hospital survival; however, there was a $22.5 \%$ greater risk of bleeding among patients receiving therapeutic anticoagulation $(3.1 \%$ vs. $2.4 \%)^{5,6}$

Analyzing patient outcomes with moderate COVID-19 (non-critically ill), it was determined that a marginal benefit of anticoagulation with heparin exists toward increased organ support-free days as well as survival when compared to thromboprophylaxis; however, among the patients receiving therapeutic anticoagulation, there was an increase of $52.6 \%$ of major bleeding risk $(1.9 \%$ vs. $0.9 \%){ }^{7}$

A recent preprint of the RAPID trial compared therapeutic anticoagulation $(N=228)$ vs. prophylactic doses $(N=237)$ in moderately ill patients with COVID-19. Mortality occurred in $4(1.8 \%)$ patients assigned to therapeutic heparin vs. $18(7.6 \%)$ assigned to prophylactic heparin (OR $0.22 ; 95 \% \mathrm{CI}, 0.07$ to $0.65)$. The risk of bleeding was not significant. ${ }^{8}$

Caution is advised against therapeutic anticoagulation in critically ill patients with COVID-19 who have no evidence of VTE given their significant predisposition to suffer major bleeding. The COVID-19 pandemic has created a concerning pattern where evidence-based medicine and established guidelines are sometimes neglected in favor of imaging protocols and therapeutic decisions contrary to a reasonable standard of care. We recommend a personalized approach in the decision to use anticoagulation in such cases, being mindful of sometimes marginal benefits weighed against the documented risks as noted.

Moises Auron, MD, FAAP, FACP, SFHM ${ }^{1}$

Mateo Porres-Aguilar, MD, FACP, CACP

Scott J. Cameron, MD, PhD, RPVI, FSVM, FACC $3^{3}$

${ }^{1}$ Department of Hospital Medicine, Cleveland Clinic, Cleveland, $\mathrm{OH}$, USA

${ }^{2}$ Division of Hospital Medicine, Department of Internal Medicine, Texas Tech University Health Sciences Center,

El Paso, TX, USA 
${ }^{3}$ Department of Cardiovascular Medicine, Cleveland Clinic,

\section{Cleveland, $\mathrm{OH}$, USA}

Corresponding Author: Moises Auron, MD, FAAP, FACP, SFHM1; Department of Hospital Medicine, Cleveland Clinic, Cleveland, $\mathrm{OH}$, USA (e-mail: auronm@ccf.org).

\section{REFERENCES}

1. García-Cervera, C., Giner-Galvañ, V., Wikman-Jorgensen, P. et al. Estimation of Admission D-dimer Cut-off Value to Predict Venous Thrombotic Events in Hospitalized COVID-19 Patients: Analysis of the SEMI-COVID19 Registry. J. Gen. Intern. Med. (2021). https://doi.org/10.1007/ s11606-021-07017-8

2. Elbadawi A, Elgendy IY, Sahai A, Bhandari R, McCarthy M, Gomes M, Bishop GJ, Bartholomew JR, Kapadia S, Cameron SJ. Incidence and Outcomes of Thrombotic Events in Symptomatic Patients With COVID-19. Arterioscler. Thromb. Vasc. Biol. 2021;41(1):545-547.

3. Weinberg I, Fernández-Capitán C, Quintana-Díaz M, et al. Systematic testing for venous thromboembolism in hospitalized patients with COVID-
19 and raised D-dimer levels. Thrombosis Update. 2021;2:100029. https://doi.org/10.1016/j.tru.2020.100029.

4. Patel NG, Bhasin A, Feinglass JM, Angarone MP, Cohen ER, Barsuk JH. Mortality, critical illness, and mechanical ventilation among hospitalized patients with COVID-19 on therapeutic anticoagulants. Thrombosis Update. 2021;2:100027. https://doi.org/10.1016/j.tru.2020.100027.

5. Therapeutic anticoagulation with heparin in critically ill patients with COVID-19. The ATTACC, ACTIV-4a, and REMAP-CAP Investigators. N Engl J Med 2021; DOI: Published ahead of print (August 4, 2021) 10.1056/ NEJMoa2103417

6. Leentjens J, van Haaps TF, Wessels PF, Schutgens REG, Middeldorp S. COVID-19-associated coagulopathy and antithrombotic agents-lessons after 1 year. Lancet Haematol. 2021 Jul;8(7):e524-e533.

7. Therapeutic anticoagulation with heparin in non-critically ill patients with COVID-19. The ATTACC, ACTIV-4a, and REMAP-CAP Investigators. N Engl J Med 2021; DOI: Published ahead of print (August 4, 2021) 10.1056/ NEJMoa2105911

8. Sholzberg M, Tang GH, Rahhal H, et al. RAPID Trial investigators. Heparin for Moderately Ill Patients with Covid-19. medRxiv 2021.07.08.21259351. https://doi.org/10.1101/2021.07.08.21259351.

Publisher's Note: Springer Nature remains neutral with regard to jurisdictional claims in published maps and institutional affiliations. 